\title{
DIFFERENT MODALITIES OF RECONSTRUCTION IN FOURNIER GANGRENE
}

\section{Moustafa Mohamed El Saady*MD, Samy Khalifa Ahmed*MD, Raafat A. El latif Mansour *MD, Fady Mehany Habib Salamh *M.Sc.}

General Surgery department, Faculty of Medicine, Zagazig University

\begin{abstract}
Background: Fournier gangrene is an acute and potentially lethal necrotising fasciitis that involves the scrotum and perineum. This disease can result in the loss of skin and soft tissue. Multiple options for reconstruction of the scrotum are available. To repair the scrotal and perineal defects remains a surgical challenge. Objective: The aim of this work is to compare different modalities of reconstruction in Fournier gangrene and to verify the indications of efficacy of each modality. Patients and methods: The present study includes 20 patients with diagnosis of Fournier gangrene from the General Surgery Departments, Faculty of Medicine, Zagazig University Hospitals from 2010 to 2012. Following admission, correction of the fluid and electrolyte imbalance, and estimation of the extension and depth of the gangrenous area were done. After stabilization of the patients, all patients underwent surgical debridement with frequent dressing, and then the patients were divided randomally into four groups according to the reconstructive procedure. Results: A total of 20 cases with Fournier gangrene were included in this study. All patients included in this study were males. Their age ranged from 20-70 years old with a mean age of 50 years. The most common comorbid condition was diabetes mellitus in $50 \%$ of cases. The infection was polymicrobial in $70 \%$ of cases. Wound healing with secondary repair was performed in 5 patients. Wound healing with primary closure was performed in 5 patients. Wound healing with split-thickness skin graft was performed in 5 patients. Wound healing with gracilis muscle flap followed by splitthickness skin graft was performed in 5 patients. No surgical mortality was recorded. Conclusion: Gracilis muscle flap plus split-thickness skin graft covered the deep perineal defects very well. It is a reliable procedure, which provides excellent cover for the exposed testes and gives satisfactory protection and aesthetic outcome.
\end{abstract}

\section{INTRODUCTION}

F ournier gangrene is a rare, rapidly progressive, fulminant form of necrotizing fascitis of the genital, perianal and perineal regions, which may extend up to the abdominal wall between the fascial planes ${ }^{[1]}$. This polymicrobial infection, which is caused by both aerobic and anaerobic bacteria, leads to thrombosis of subcutaneous and cutaneous blood vessels, resulting in sever gangrene of the overlying $\operatorname{skin}^{[2]}$.

It is believed that systemic conditions such as diabetes mellitus, alcholism, paralysis or neurological deficit, malignancy, debilitated and immunosuppressed individuals are predisposed to the development of this entity ${ }^{[3]}$.

The treatement of choice for Fournier gangrene is aggressive surgical debridement. Scrotal reconstruction is challenging. Function, cosmosis and psychology of the patient have to be considered $^{[4]}$.

Primary suturing is often described for small defects. Larger defects might require usage of skin grafts. Soft tissue flaps as gracilis muscle flap provide excellent cover for the exposed testes and give satisfactory protection and aesthetic outcome [5]

Aim of the work: The aim of this work is to compare different modalities of reconstruction in Fournier gangrene and to verify the indications of efficacy of each modality.

Patients and methods: The present study includes 20 patients admitted to Zagazig University Hospital in Emergency unit of General
Surgery Department with the diagnosis of Fournier gangrene during the period from 2010 to 2012. Following admission, correction of the fluid and electrolyte imbalance, and estimation of the extension and depth of the gangrenous area were done.

All patients were subjected to the following:

\section{I- Preoperative evaluation:}

1- Medical history taking with stress on age, gender, onset of symptoms, course of disease and duration of symptoms

2- Past medical and surgical history including diabetes mellitus, alcoholism, and previous trauma or surgery.

3- The time between the onset of the disease and reference to the medical center.

4-Routine pre-operative investigations.

5- Wide-spectrum antibiotic therapy had been initiated empirically before the results of tissue culture were obtained. This empiric therapy included the combination of a $3^{\text {rd }}$ generation cephalosporin, an aminoglycoside, and metronidazole or clindamycin. Then, antibiotic therapy was changed according to the results of tissue culture and sensitivity.

6- Imaging studies as scrotal ultrasound and CT scan.

\section{II-operation:}

- $\quad$ Stage of debridement: (Figure 2) Early and aggressive surgical debridement is essential, because it significantly decreases morbidity and mortality. The aim of debridement was to remove the origin of the infection as well as the infected 
tissues. After debridement of necrotic tissues was done, the testis was left exposed.

- Following surgical debridement of necrotic tissues, the wound was allowed for additional chemical debridement to prevent reinfection and to promote natural healing and granulation. Hydrogen peroxide, Eusol, povidone iodine, and saline solution were the agents most often used.

- Stage of reconstruction: Once the patient is stable and in an anabolic state with granulating wounds, reconstruction of the denuded areas can be done.

- $\quad$ The patients are divided into four groups according to method of reconstruction:

Group (A) Wound healing with secondary repair. Group (B) (Figure 3) wound healing with primary closure.

Group (C) (Figure 4) wound covered with skin grafts.

Group (D) (Figure 5) wound covered with gracilis muscle flap followed by skin graft.

\section{III-postoperative care and follow up:}

The patients would be followed up in the early postoperative period for - The hospital stay.

- The presence of infection.

- Postoperative pain and bleeding.

- Viability of the flap.

- Take of the skin graft.

All patients would be followed up monthly for 6 months for the cosmetic appearance and bulkiness of the flap.

Results:

Demographic data of the studied patients: (Table: 1) (Figure: 1) Twenty patients with Fournier gangrene were included in the present study. All patients included in this study were male patients. Their age ranged from 20-70 years old with a mean age of $50 \pm 10.1$ years.

\section{Predisposing Factors:}

From a total number of 20 cases, it was found that 10 patients $(50 \%)$ had FG secondary to anorectal conditions - perineal abscess (6), ischiorectal abscess (2), and posthaemorrhoidectomy (2). No etiologic factors for FG were found in 5 (25\%) patients and they were classified as idiopathic FG. Five patients $(25 \%)$ developed FG secondary to urogenital infections (scrotal abscess in four patients and one following scrotal trauma).

Comorbidities: (Table: 2) Some diseases predisposed to Fournier gangrene. The most frequent pre-existing comorbidities were diabetes mellitus in 10 patients $(50 \%)$, chronic liver disease in 5 patients (25\%), malignancy and chemotherapy in 2 patients $(10 \%)$, cardiac disorders in 1 patient $(5 \%)$, renal failure in 1 patient (5\%), and no definite predisposing factor in 1 patient (5\%).

Clinical signs: The clinical presentation of the selected patients was quiet variable. 16 patients presented with oedema (80\%), 14 patients presented with erythema (70\%), 18 patients presented with necrosis (90\%), 16 patients presented with discharge (80\%), 12 patients presented with crepitance $(60 \%)$ and 8 patients presented with fluctuance (40\%).

Location of lesion: (Table: 3) Among the selected patients, the lesion involved the penile shaft in 16 cases $(80 \%)$, the scrotum in 20 cases $(100 \%)$, the perineum in 10 cases $(50 \%)$, the suprapubic region in 5 cases $(25 \%)$, the inguinal region in 5 cases $(25 \%)$ and the urethra in 1 case (5\%).

\section{Investigations:}

1. Scrotal ultrasound was done for all patients showing thickened, oedematous scrotal wall with normal size and vascularity of testes and epididymis due to their separate blood supply. The thickened scrotal wall shows hyperechoic foci that demonstrate reverberation artifacts; causing "dirty" shadowing that represents gas within the scrotal wall in 12 patients $(60 \%)$.

2. CT scan was done for all patients showed softtissue thickening and inflammation. Subcutaneous emphysema secondary to gas-forming bacteria was found in 12 cases $(60 \%)$. CT scan also demonstrated the underlying cause as perianal abscess in 6 cases (30\%), ischiorectal abscess in 2 cases $(10 \%)$, and scrotal abscess in 4 cases $(20 \%)$.

\section{Operative results:}

1- Debridement: (Figure: 2) Surgical debridement was performed once on 8 patients, twice on 6 patients, three times on four patients and four times on one patient. The number of surgical debridements did not seem to influence patient outcome.

2- Reconstruction: The 4 groups mentioned before are compared according to:

Operative time: The operative time for group (B) patients ranged from 30-60 minutes with a mean of 45 minutes, while in group (C) patients the operative time ranged from 60- 90 minutes with a mean of 75 minutes. In group (D) patients the operative time ranged from 110-155 minutes with a mean of 125 minutes.

The need for blood transfusion: In the 4 groups of patients included in this study, there were no significant operative blood losses that necessitate blood transfusion intraoperative.

\section{Early postoperative results}

Postoperative hospital stay:

(Figure: 7)

Postoperative follow up of the studied patients showed that hospital stay was variable between 
the 4 groups. In group (A) the mean hospital stay was 20 days (range: 15-25 days), in group (B) the mean hospital stay was reduced to 5 days (range: 3-7 days), in group (C) the mean hospital stay was 8.4 days (range: 7-13 days), while in group (D) the mean hospital stay was 12.5 days (range: 1015 days).

Postoperative Infection:

(Table: 4) Postoperative infection was noted in 2 patients of group (A) managed conservatively by dressing. Only one patient of group (B) had wound infection resulting in wound dehiscence and managed by repeated dressing then reapproximation by suturing. One patient of group (C) developed wound infection resulted in partial graft loss that was managed by frequent dressing. One patient of group (D) developed superficial muscle infection that necessitates superficial debridement and dressing.

\section{Late postoperative results}

On follow up of the 4 groups of patients included in this study we have found that the size of scrotum was reduced in groups $\mathrm{A}, \mathrm{B}$ and $\mathrm{C}$ while in group $\mathrm{D}$ the size of the scrotum is not significantly reduced with satisfactory aesthetic outcome to both surgeon and patient.

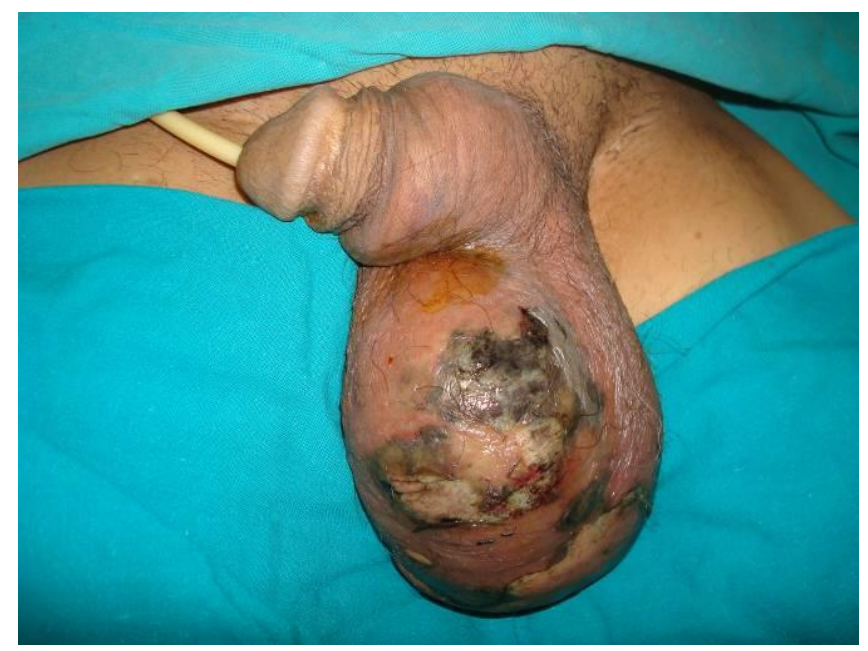

Figure (1) : Fournier' gangrene in 67 years male patient

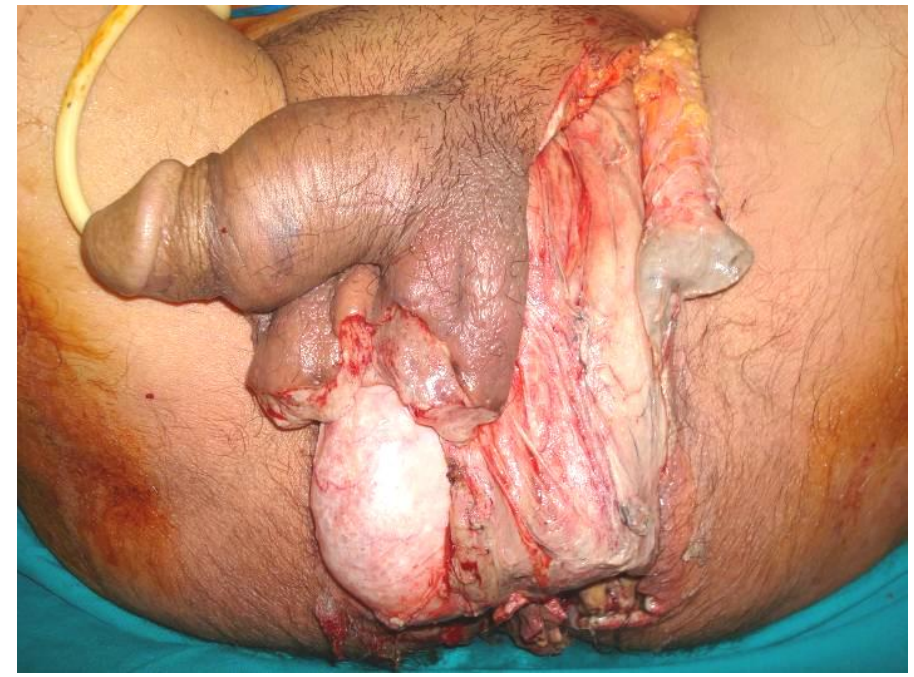

Figure (2): Fournier's gangrene after one setting of debridement 


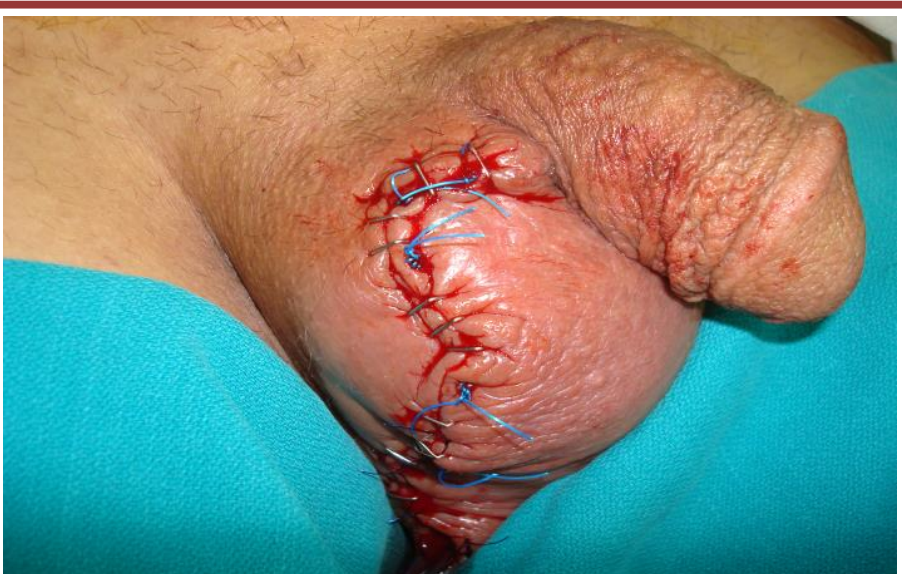

Figure (3): Fournier gangrene closed by secondary suture

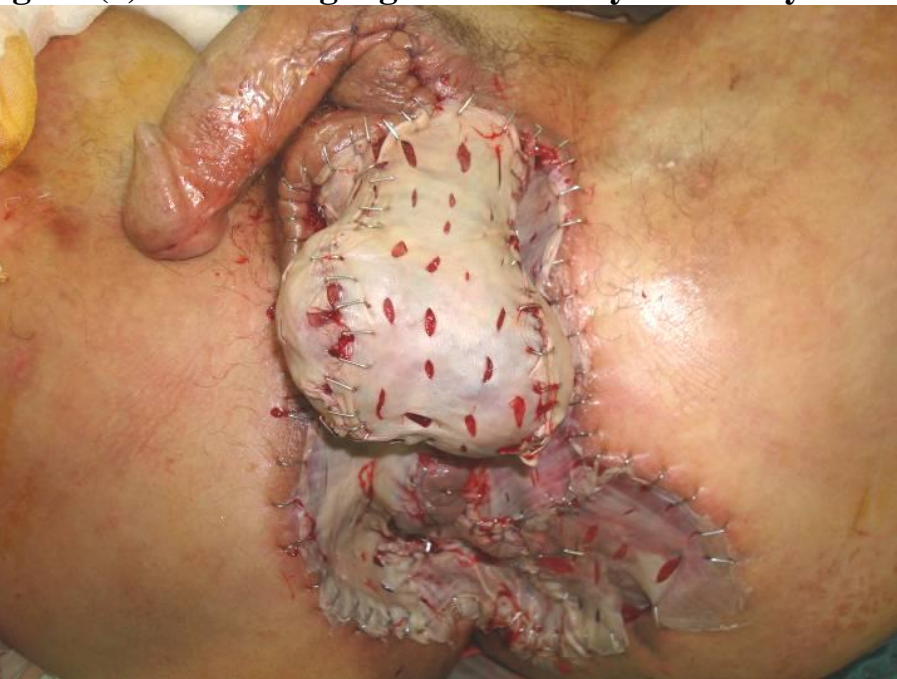

Figure (4): Fournier gangrene reconstructed by skin graft.

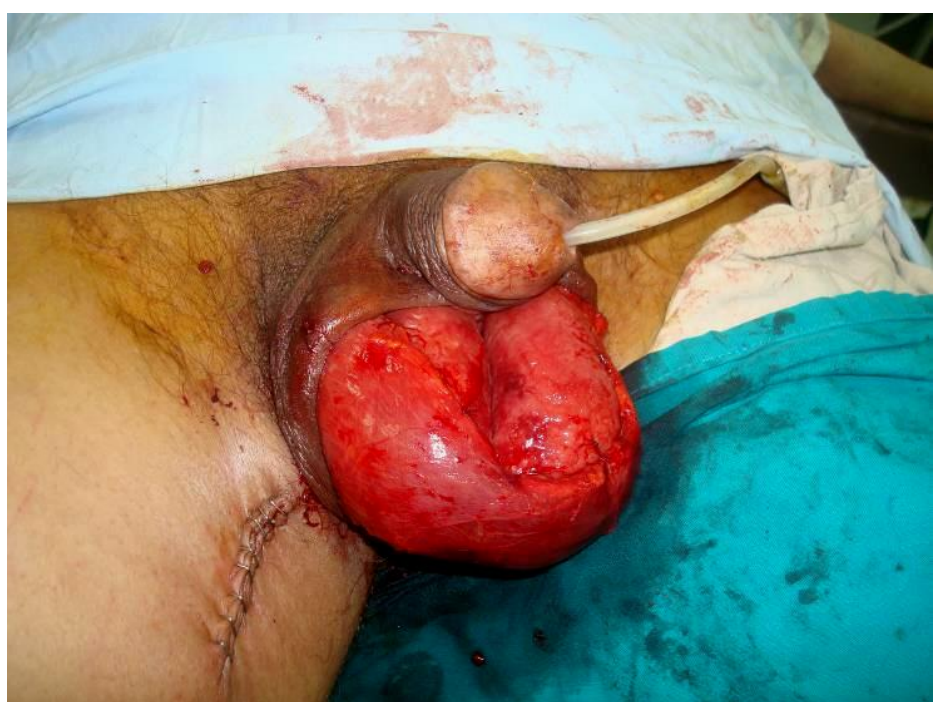

Figure (5): Fournier gangrene reconstructed by gracilis muscle flap. 


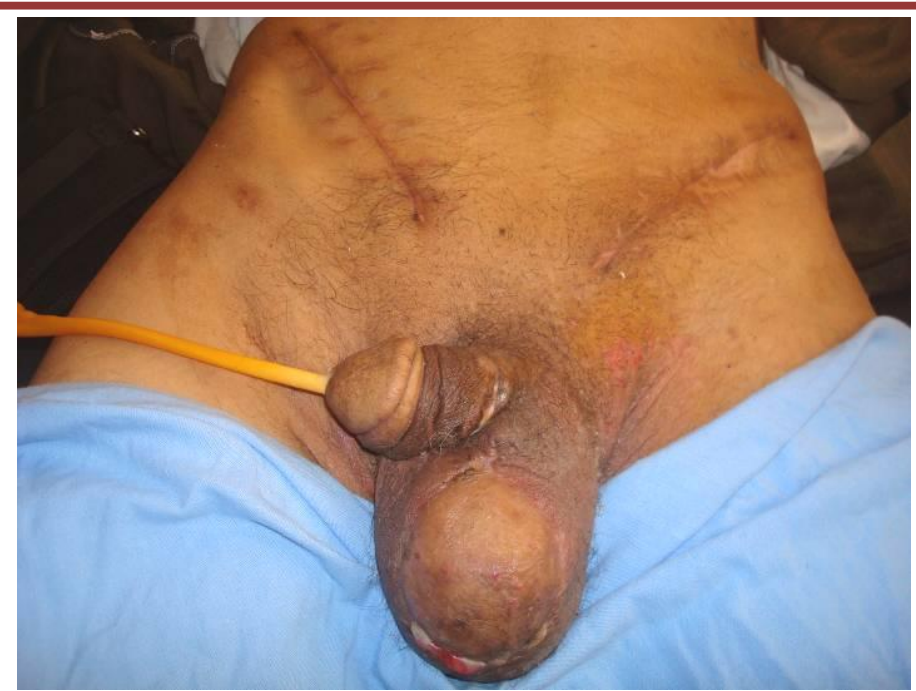

Figure (6): Fournier gangrene reconstructed by gracilis flap and skin graft (late postoperative view).

Table (I) : The age characters of the studied patients.

\begin{tabular}{llll}
\hline & Age / years & Number & Percent \\
\hline $20-30$ & 1 & $5 \%$ \\
\hline $31-40$ & 3 & $15 \%$ \\
\hline $41-50$ & 7 & $35 \%$ \\
\hline $51-70$ & 9 & $40 \%$ \\
\hline Total & 20 & $100 \%$ \\
\hline
\end{tabular}

Table (II) : The distribution of patients according to Co-morbid factors.

\begin{tabular}{lll}
\hline Co-morbidity & Number & Percent (\%) \\
\hline Diabetes mellitus & 10 & $50 \%$ \\
\hline Chronic liver disease & 5 & $25 \%$ \\
\hline Malignancy and chemotherapy & 2 & $10 \%$ \\
\hline Cardiac disorders & 1 & $5 \%$ \\
\hline Renal failure & 1 & $5 \%$ \\
\hline Undefined & 1 & $5 \%$ \\
\hline
\end{tabular}

Table (III): The distribution of patients according to location of lesion

\begin{tabular}{lll}
\hline Location of lesion & Number & Percent (\%) \\
\hline Penile shaft & 16 & $80 \%$ \\
\hline Scrotum & 20 & $100 \%$ \\
\hline Perineum & 10 & $50 \%$ \\
\hline Suprapubic & 5 & $25 \%$ \\
\hline Urethra & 1 & $5 \%$ \\
\hline
\end{tabular}




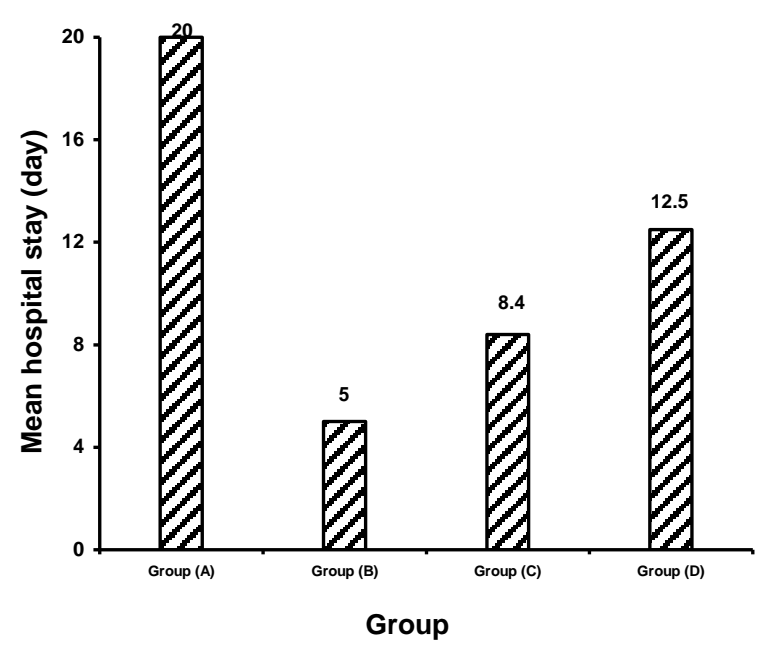

Figure (7): The distribution of patients according to Postoperative hospital stay Table (IV): The distribution of patients according to Postoperative infection.

\begin{tabular}{ccc}
\hline Groups & Number of patients & Percent (\%) \\
\hline Group (A) & 2 & $40 \%$ \\
\hline Group (B) & 1 & $20 \%$ \\
\hline Group (C) & 1 & $20 \%$ \\
\hline Group (D) & 1 & $20 \%$ \\
\hline
\end{tabular}

\section{DISCUSSION}

Fournier gangrene is an acute idiopathic gangrene frequently of insidious onset followed by a fulminant progression that may lead to significant morbidity or even mortality. Although occasionally limited in extent, it is more frequently associated with significant loss of the skin of the penis, scrotum and surrounding perineum ${ }^{[5]}$.

The common routes of bacterial entry in Fournier gangrene include urinary tract infection, peri-anal infection or local trauma to the scrotum and/or perineum. Patients, who have medical illnesses such as diabetes, cirrhosis of the liver, alcoholism, uraemia, malignancy, colorectal infection or urological disease, are at higher risk of this disease. Diabetes mellitus is the most common predisposing factor for Fournier gangrene ${ }^{[6]}$.

It is important to recognize that Fournier gangrene is a progressive disease and can spread rapidly to cause extended necrosis of the skin and underlying soft tissue, and that it has a high mortality rate. Early diagnosis, use of broadspectrum antibiotics and timely surgical debridement are critical factors for treating Fournier gangrene successfully and increasing the survival rate ${ }^{[7]}$.

The disease now differs from its original description (of 1883) in that it now affects both genders and is no more idiopathic in the majority of cases but in our study all the patients were male [8].

A majority of the patients belong to the older age group as also found in our study. The interval from onset of clinical symptoms to the initial surgical intervention appears to be the most important prognostic factor with a significant impact on outcome ${ }^{[9]}$.

A typical patient presents 2-7 days after initiation of symptoms. Late presentation was evident in our patients, possibly due to social taboos causing reluctance to examination of the perineal/genital regions ${ }^{[8]}$.

It is crucial to excise the necrotic soft tissue including skin, subcutaneous fat and fascia during the surgical debridement and to preserve as much of the normal tissue as possible. After the first debridement, wound care with wet-gauze dressing and frequent checks of the wound condition are helpful. Additional surgical debridement is desirable when the wound base shows residual dead tissue ${ }^{[9]}$.

After sufficient treatment, the wound base showed healthy granulation tissue, and reconstructive surgery for skin and soft-tissue coverage could be considered at this stage ${ }^{[10]}$.

Reconstruction of the scrotum after complete loss of the overlying skin is a challenging problem for 
the reconstructive surgeon. Reconstructing the scrotum is essential not only for cosmetic reasons but also for functional and psychological reasons [12].

The best functional and cosmetic results for scrotal reconstruction after Fournier gangrene can be achieved with primary closure of any remaining scrotum without undue tension. This is, however only possible with small defects. If the defect is too large to be closed primarily, it should not be allowed to close by secondary intention since this would not only prolong the healing time but will also lead to contraction and deformity of the scrotum ${ }^{[13]}$.

With complete loss of the scrotum another option is needed to cover the testicles. Coverage has to be achieved in a way that will restore function quickly and provide good cosmetic outcome with low associated morbidity and mortality ${ }^{[8]}$.

Fournier's gangrene often occurs in patients with advanced age, diabetes, alcoholism and other preexisting medical problems, which may increase their operative risk, particularly for longer or multiple procedures. The ideal method of coverage should be technically easy to perform and of low cost ${ }^{[14]}$.

Several techniques for coverage of the testicles have been described. A simple and widely used method of treatment has been placement of the testes in subcutaneous pockets in the thighs. This technique offers quick coverage of the testes but has several drawbacks. The main aesthetic and psychological problem is the absence of the scrotum. Functionally, the placement of the testes in the thighs can cause discomfort while walking or stretching. Some authors have used this technique as a first stage coverage for later reconstruction with flaps ${ }^{[8]}$.

Split thickness skin graft of scrotal defects is simple and can be performed in one stage. The results are cosmetically and functionally satisfactory. The color and shape as well as the thickness of the skin on the neoscrotum are close to normal. The thin skin can keep the testicles cool, imitating the environment provided by normal scrotal skin ${ }^{[14]}$.

However, there are some disadvantages of splitthickness skin graft for reconstruction of scrotal and perineal defects. The appearance is not completely natural, since the new sac lacks redundant skin and the testicles are not freely floating. Instead, the testicles will remain in a low position due to loss of cremasteric function ${ }^{[10]}$.

The graft may not take adequately when applying it on an uneven or concave wound surface. In addition, a split-thickness skin graft is not as durable as a fasciocutaneous or myocutaneous flaps in protecting from vulnerability to damage [15].

We applied split-thickness skin grafting in five patients and recommend this technique when most of the defect area involves the flat surface of the lower abdominal wall. If the skin defects combine a large and deep pocket, a muscle flap is needed to eliminate the dead space ${ }^{[16]}$.

Numerous thigh cutaneous or fasciocutaneous flaps have been designed for primary or secondary scrotal repair when the testicles have already been temporarily buried under thigh skin ${ }^{[5]}$.

The gracilis muscle, which can be harvested as a muscle flap or a myocutaneous flap, is a good candidate to fill the deep perineal defects. It is a broad sheet like muscle that can be wrapped easily around the acute curves of the exposed testes and do not add much bulk to the reconstructed scrotum $^{[16]}$.

We performed gracilis muscle flap plus splitthickness skin graft in five patients. Gracilis muscle flap covered the deep perineal defects very well. It is simple and reliable procedure, which provides excellent cover for the exposed testes and gives satisfactory protection and aesthetic outcome. This made it recommended to use for primary reconstruction of major scrotal defects following Fournier gangrene $^{[17]}$.

\section{CONCLUSION}

The best functional and cosmetic results for scrotal reconstruction after Fournier gangrene can be achieved with primary closure of any remaining scrotum without undue tension. This is, however only possible with small defects.

Split thickness skin graft of scrotal defects is simple and can be performed in one stage. The results are cosmetically and functionally satisfactory. However, there are some disadvantages of split-thickness skin graft, the appearance is not completely natural, since the new sac lacks redundant skin and the testicles are not freely floating. Instead, the testicles will remain in a low position due to loss of cremasteric function.

Gracilis muscle flap covered the deep perineal defects very well. It is a reliable procedure, which provides excellent cover for the exposed testes and gives satisfactory protection and aesthetic outcome. This made it recommended to use for primary reconstruction of major scrotal defects following Fournier gangrene.

\section{REFERENCES}

[1] Sahin K, Mehmet Y and Faik Y (2008): The clinical features of Fournier gangrene. Int. Urol. Nephrol., 40: 997-1004. 
[2] Atil C, Volkan G and Cihangir A (2008): Fournier gangrene, is it scrotal gangrene? Adv. Ther., 25(10): 1065-74.

[3] Ghnnam WM (2008): Fournier gangrene in Mansoura Egypt. J. Postgrad. Med., 54: 106-9.

[4] Sevin K, Emiroglu M and Yormuk E (2008): Different modalities of scrotal reconstruction. Eur. J. Plast. Surg., 15: 303-305.

[5] Hallock GG (2006). Scrotal reconstruction following Fournier gangrene using the medial circumflex femoral artery perforator flap. Am. Plast Surg ., 57(3): 333-35.

[6] Yanar H, Taviloglu K and Ertekin C (2006). Fournier gangrene: risk factors and strategies for management. World J Surg., 30: 1750-4.

[7] Ersay A, Yilmaz G and Akgun Y (2007). Factors affecting mortality of Fournier gangrene: review of 70 patients. ANZ J Surg., 77:43-8.

[8] Norton K, Johnson L, Perry T, Perry K, Sehon J and Zibari G (2002). Management of Fournier gangrene: an 11-year retrospective analysis of early recognition, diagnosis, and treatment. An Surg., 68:709-13.

[9] Korkut M, Icoz G, Dayangac M, Akgun E, Yeniay L, Erdogan O and Cal C (2011). Outcome analysis in patients with fournier gangrene: report of 45 cases. Dis. Colon Recum., 83: 649-52.

[10] Czymek R, Schmidt A and Eckmann C (2009). Fournier gangrene: vacuum-assisted closure versus conventional dressings. Am J Surg., 197:168-76.
[11] Ferreira P.C., Reis J.C., Amarante J.M., Silva Á.C., Pinho C.J., Oliveira I.C and da Silva P.N (2007). Fournier Gangrene: A Review of 43 Reconstructive Cases. Plast. Reconstr. Surg., 119 (1): 175-184.

[12] Mohammed G. Ellabban and Paul L. Townsend (2003). Single-stage muscle flap reconstruction of major scrotal defects: report of two cases. The British Association of Plastic Surgeons., 56, 489493.

[13] Campbell JC (1955). Fournier gangrene. $\mathrm{Br} \mathrm{J}$ urol., 27: 106-13.

[14] Pirko Maguiña, Tina L. Palmieri and David G. Greenhalgh (2003). Split thickness skin grafting for recreation of the scrotum following Fournier gangrene. Burns., 29: 857-862.

[15] Shih-Yi Chen, Ju-Peng Fu, Tim-Mo Chen and Shyi-Gen Chen (2011). Reconstruction of scrotal and perineal defects in Fournier gangrene. Journal of Plastic, Reconstructive \& Aesthetic Surgery., 64: 528-534.

[16] Kayikcioglu A (2003). A new technique in scrotal reconstruction: short gracilis flap. Urology., 61:1254-6.

[17] Calderon W, Chang N and Mathes SJ (2010). Comparison of the effect of bacterial inoculation in musculocutaneous and fasciocutaneous flaps. Plast Reconstr Surg., 135:785-92. 